\title{
The problems of monitoring the deformation processes in the integrated development of the underground space of metropolitan cities
}

\author{
Dmitriy Neguritsa ${ }^{* 1}$ \\ ${ }^{1}$ Federal State Autonomous Educational Institution of Higher Education "Peoples 'Friendship \\ University of Russia" (RUDN), Engineering Academy of Peoples' Friendship University of Russia, \\ Moscow, Russia
}

\begin{abstract}
By analyzing the existing regulatory documents regulating the surveying and geodetic monitoring of buildings and structures from the negative impact of mining and construction works, conclusions were drawn about the problems of its implementation in the conditions of modern metropolitan cities and the imperfection of the methodology for observing deformational processes in the construction of underground structures. New approaches to the organization of monitoring of deformation processes in metropolitan cities were proposed.
\end{abstract}

\section{Introduction}

Despite the crisis phenomena in the economy, modern metropolitan cities are developing dynamically. One of the main trends is the complexity of development: the increase in the number of storeys, the development of underground space, the development of transport above-ground, ground and underground infrastructure, the subway, the construction of various communications [1-3].

Currently, the territory of Moscow is overloaded with objects influencing change in engineering geological and geotechnical conditions in the city. Due to urbanization and consequently, the acceleration of human impact, the increase in the geomechanical load on the massif and the degradation of its bearing capacity takes place [4].

\section{Factors affecting the development of deformation processes in the development of underground space}

Underground space of cities is a valuable georesource, the cost, resource intensity and development efficiency of which is determined by its geomechanical, geotechnical properties, geosituational features and the dynamics of their changes in the development.

In the modern city-planning situation, when underground structures and communications are being built, buildings and structures are being erected, their geomechanical processes are being reconstructed, having different directions, the time of

*Corresponding author: neguritsa_dl@rudn.university 
passage and the intensity affect a large number of objects of different responsibility levels. This leads to deformation of existing buildings and structures falling into the zone of influence, varying degrees of their damage and even to the possible occurrence of emergencies [5-7].

The study of the causes of accidents and deformations of buildings and structures shows that in most cases they occur not because of errors in engineering calculations, design, installation of above-ground structures, but due to miscalculations made during engineering and geological surveys, design and construction of foundations and casting concrete, incorrect operation of buildings, leading to a deterioration in the properties of soils in the foundation, as well as due to insufficient account of the joint influence of several underground structures.

Therefore, to control the safe flow of geomechanical processes and the forecast of their development, it is necessary to take into account all the factors that influence the development of deformation processes [8].

At the moment, factors that influence the development of deformations are well studied, methods for forecasting and controlling their development and calculation methods have been developed, there exists an instrument base for measuring displacement and deformation, but from the engineering point of view there is a lack of implementation of an unambiguous and effective solution to this problem.

This is due to a number of reasons, namely:

-the heterogeneous structure of the soil-rock massif and the variability of its properties at different degrees of intensity of the impact thereon;

- the complexity of describing deformation processes due to the influence of various sources and the uneven spread of their influence in different parts and in different directions;

- Nonlinearity of development of deformation processes in the soil-rock massif;

- Mutual influence of structures with dynamic loads and passive structures and communications;

- The impossibility to know the true state (degree of wear and safety factor) for a large number of structures and communications;

- The development of an emergency situation so quickly that the use of preventive and protective measures is impossible;

- The discrete development of deformation processes in time and space, which can be the reason for their uncontrolled development.

Emergency situations on objects usually arise not under the influence of one factor, but when combined and added together, including a certain sequence of their influence.

At the same time, there is some inconsistency between the current regulatory documents on monitoring, ensuring industrial safety and the methodology for their application in urban underground and surface construction, since under certain conditions the development of geodynamic processes can be instant and irreversible, which completely excludes the use of protective or preventive measures.

\section{Principles of geomonitoring organization}

To date, a fairly large number of methods for monitoring deformation processes have been developed, but the most effective ones in terms of accuracy - reliability - efficiency cost are the geodetic and mine surveying methods that provide high accuracy, efficiency and reliability.

Existing methods of forecasting the expected shifts and deformations, as a rule, allow obtaining their greatest values. 
To control the development of deformation processes in the process of construction and operation of an underground structure, geomechanical monitoring is performed. Geomechanical monitoring is a complex of measures to control the development of deformation processes performed under a special project whose main task is to identify the signs preceding the creation of emergency situations.

Geomechanical monitoring is carried out under a special project, which is an integral part of design documentation and undergoes industrial safety expertise.

The geomechanical monitoring project consists of the following sections:

- Geological and geomechanical description of construction conditions;

- Technological description of the process of erection of an underground structure;

- Forecast of development of deformation processes;

- Examination and determination of the maximum and permissible deformations of buildings and structures falling into the zone of the construction influence;

- the definition of a set of protective and preventive measures that must be applied to individual buildings and structures for which allowable and limiting values of deformations are less than predicted ones;

- The description of the structures of observation stations, the construction of reference points, the methods of their laying;

- The description of applied measuring devices and the equipment;

- The procedure of measurement and processing of received data;

- The analysis of the obtained measurement data, comparison of measured values of shifts and deformations with permissible and limiting values;

- The preparation of a report on the development of deformation processes;

- Making expert decisions on the protection of objects, buildings and structures.

When performing geomechanical monitoring, the most important stages are the stage of forecasting the development of deformation processes and the stage of monitoring the development of displacement processes.

Determining the zone of influence of construction and the forecast of deformation development is one of the most complex, time-consuming and responsible parts of the project since geomonitoring is organized based on this data.

For today the Russian Federation lacks a uniform forecasting technique. The existing regulatory framework lags behind the development of construction technology and does not take into account the specifics of modern construction and technologies applied. Thus, for example, the Instruction on Observations of Earth Surface Displacements and Objects Located on It in the Construction of Underground Structures in Moscow, M .: 1997 was published more than 20 years ago and does not reflect the current trends in the development of the underground space of the city. The forecast methods used in the construction industry using the finite element method and the adoption of an elastic or elasto-plastic medium do not provide sufficient accuracy since the soil stratum containing the underground structure consists of soils that are very heterogeneous in their geological composition and physico-mechanical properties. When preparing the forecast, it is necessary to take into account a large number of influencing factors that can be conditionally divided into four groups: geological, technological, geomechanical and geosituational.

The additional risk of occurrence of emergency situations is caused by the fact that the impact on other underground facilities, in particular, those that are below the constructed ones - tunnel and station buildings of the subway, etc. are often didn't take into account

The next stage after the forecast of the geomechanical monitoring is the design and laying of observation stations and the performance of observations.

The laying of observation stations and the production of observations in the city are complicated by the following: the surrounding areas are almost completely asphalted, 
except for small lawns, the existing building is very dense, in the areas of construction there is very dense pedestrian and vehicular traffic, which can significantly complicate the use of modern high-performance equipment, tacheometers with servo drive, working in automatic mode and laser scanners. In the case of particularly important buildings and structures, for example, monuments of culture, permanent monitoring systems are being installed, and it is necessary to install not one but several instruments.

One of the problems with observing the shifts is to ensure the accuracy and reliability of the obtained measured values of the shifts and the actual course of the process, taking into account that in some cases the accuracy of the measurements can be compared with the measurement values [9].

The task of ensuring the stability of the position of the initial reference points in each of the cycles is solved by laying a clump of at least three initial reference points, whose position control is performed with each observation cycle.

In modern conditions, to ensure the effectiveness of measurement work, it is necessary to constantly improve the design of benchmarks to ensure their continuous service during both the construction and also the operation of the underground facility. When laying the observation stations, a large number of benchmarks are used, having a different design, depending on the laying location destination and the service life. Usually three types of signs - basic, intermediate and deformation - are used at observation objects.

The main reference points are the reference points of the city geodetic network and are included in the main trunk way to monitor their mutual position. Some of them are located outside the zone of possible deformations from the production of construction, installation and mining works, so as to avoid the influence of settlings from the structure being built on the measurements made.

Intermediate signs serve to organize the optimal scheme for transferring the mark from the initial benchmark to the deformation one.

Deformational benchmarks are simultaneously points for determining displacements in plan and height and are fixed in such a way that their location and quantity allows revealing possible deformations. As deformation marks, the reinforcing bars laid at the base of the buildings and along the profile lines in the adjacent ground mass are used, at the end of which there is a hemispherical head for the installation of the rail and reflector.

The largest number of benchmarks is laid directly into the walls of those buildings and structures that fall into the zone of influence of construction. Deformational benchmarks are laid along the perimeter of buildings at a distance of 5-20 meters, with mandatory installation on the corners and characteristic protrusions of buildings. Marks are laid at the same height, approximately 0.5 meters from the surface of the earth. The deformation mark can have various designs. To each benchmark, a unique serial number is assigned, applied with paint on the wall of the building or a lodge at the ground bench. It is advisable to make a digital photograph of the benchmarks laid.

Measurements are made of both horizontal and vertical shifts. In the city of Moscow, horizontal shifts sometimes exceed vertical ones.

The accuracy and frequency of measurements are established by the project and is determined depending on the monitoring objectives and the conditions for the construction. Proceeding from this, the instruments and methods of measurement are selected. In complex conditions of the city, a complex method of observations with the use of various measurement technologies is often used. At the same time, settlings and horizontal shifts are measured.

In addition to measuring the shifts of objects falling into the zone of influence, measurements are made of the underground structure shifts. On the "wall in the ground", beacons are installed with a step of 5-10 $\mathrm{m}$ and horizontal and vertical movements are measured. In addition, as far as the erection and accessibility to the "wall in the ground" is 
necessary, laying benchmarks on all floors of the complex is necessary to establish the movement of the wall in the construction process. It is also necessary to observe individual structures and parts of the complex in order to establish the values of their deformation.

In the immediate vicinity of the projected complex, deep benchmarks are laid to observe the deformations of the soil massif.

The processes of shifting and deformation can develop smoothly, discretely, or move from one stage of deformation to another.

With the smooth development of the processes, observations and their processing are not problematic and the maximum reliability of the information obtained is ensured.

With the discrete development of the processes of shifting and deformation, difficulties arise both with the interpretation of the results obtained and with the determination of the origin of the local manifestation of the dynamic process. This makes it difficult to process observation data, as well as to assess the actual condition of a building or structure, which is especially important for monuments of architecture and art in modern metropolitan cities. The appearance of local dynamic manifestations of deformations alters the description of the development of deformation processes using standard techniques for processing measurement data for 3-6 benchmarks.

\section{Assessment of the accuracy of observations}

The analysis of the processes of shifting and deformation both in space and time, as well as their unevenness in one cycle of observations, is of particular importance in the processing of measurement results.

Obviously, when observing deformations of buildings and structures, it is almost impossible to determine in advance which mark will receive the maximum shifts and which areas will be deformed as much as possible.

Therefore, to calculate the accuracy, the maximum displacement standard $\sigma_{S \max }$ is determined for the weakest point of the network.

To determine the error of the settling under high-precision geometric leveling, when observing the deformations of buildings and structures in the conditions of close urban development, used in determining the vertical component of the displacement vector:

$$
S_{j i}=\left(H_{I 0}-H_{I i}\right)+\sum_{j=0}^{j} h_{j 0}-\sum_{j=0}^{j} h_{j i}
$$

where: $\left(H_{I 0}-H_{I i}\right)$ - the difference in the marks of the initial benchmark in the zero and ith cycles of observations, (the error of the change in the height of the starting point for a period of time $t_{i}$ between the zero and the current observation cycle), $\sum_{j=0}^{j} h_{j 0}$ and $\sum_{j=0}^{j} h_{j i}-$ the sum of all the excesses, respectively, in the zero and i-th cycles.

This error is summed from the sum of the error of settling or jacking of the benchmark and the change in the length of the benchmark structure under the influence of seasonal temperature fluctuations.

To reduce the negative impact of these factors, it is advisable to lay a clump of reference points, the superelevation; between which is determined with the greatest possible accuracy in these conditions to identify the most stable reference point by the criterion of stability. In practice, the Tarnowski stability criterion is used quite often:

$$
K=2 \mu_{H} \sqrt{2 n}
$$

where: $\mu_{H}$-the standard for determining elevations at a station, $n$-the number of stations between the reference points.

The range of values from $+\mathrm{K}$ to $-\mathrm{K}$ where it is impossible to detect settlings.

After the measurements, calculations of the slopes, curvatures, and horizontal deformations are performed, allowing to comprehensively evaluating the development of the shift and deformation processes. 
In the process of monitoring the shift and deformation of structures of significant height, it is necessary to pay special attention to the observations of the roll, which will allow performing accident-free construction and installation work and monitoring the development of deformation processes.

The use of a modern hardware base for measurements will make it possible to perform measurements with high efficiency, but it will require special algorithms for processing the results of measurements obtained with their help, analyzing them and, on the basis of the received data, adopting technical, technological or managerial decisions [10].

In the time frame established by the project, as well as in the event of abnormal situations, reports on the development of deformation processes and recommendations on the implementation of protective measures are prepared and sent to other interested specialists.

Using the GIS tools to analyze the development of deformation processes, it is possible to establish the parameters and the nature of deformation processes development both in space and time, which will allow not only to monitor their progress and establish the signs preceding the emergence of an emergency situation, but also to clarify the patterns of development of deformation processes in these conditions.

Measurements are made until the displacement process is completed and in the first two years of the operation of the underground complex.

\section{Conclusions}

Thus, the main goal of geomechanical monitoring is the creation of a system for observing the development of deformation processes in the soil massif and the earth's surface, which ensures the timely detection of signs preceding the emergency situations in such a way that it is possible to take the necessary preventive and protective measures in advance. For this purpose, a forecast of deformations is made, schemes of observation stations are compiled and monitoring of the state of the surface, objects and structures during the construction and installation works is carried out.

\section{References}

1. B.A. Kartoziia, A.V. Korchak, A.N. Levchenko, Mining Magazine, 9, 60-64 (2018)

2. Wout Broere, Tunnelling and Underground Space Technology, 55, 245-248 (2016).

3. Priscilla P. Nelson, Tunnelling and Underground Space Technology, 55, 32-39 (2016).

4. V.E. Merkin, D.S. Koniukhov, Underground and tunnels, 1-2, 18-23 (2017)

5. K. N. Trubetskoi, Mining Magazine, 7, 19-23 (2015).

6. K. N. Trubetskoi, M.A. Iofis, Markscheider bulletin, 4, 27-30 (2007)

7. A.Z. Vartanov, I.V. Petrov, A.V. Fedash, Mining information-analytical bulletin (scientific and technical journal), 10, 160-164 (2015)

8. E.Iu. Kulikova, A.V. Korchak, A.N. Levchenko, Risk management strategy in urban underground construction (Moscow State Mining University Publishing House, 2005)

9. D.L. Neguritsa, Problems and prospects of integrated development and conservation of the earth's interior, 175-178 (IPKON RAS, 2018)

10. D.L. Neguritsa, Kh.S. Alafar, Bulletin of Russian University of Peoples' Friendship. Series: Engineering research, 1, 22-27 (2016). 